\title{
Fernando Broncano
}

Universidad Carlos III de Madrid, España

fernando.broncano@uc3m.es

\section{Carlos Mario Muñoz-Suárez}

Universidad Icesi, Colombia

carlosmariomunozsuarez@gmail.com

\section{Cognición, tecnología y racionalidad Entrevista a Eduardo Broncano}

Cognition, Technology and Rationality Interview to Eduardo Broncano

Cognição, tecnologia e racionalidade Entrevista a Eduardo Broncano

Artículo de reflexión recibido el 01/06/2010 y aprobado el 02/11/2010 



\title{
Resumen
}

La cognición, la tecnología y la racionalidad hacen constituyen tres dominios de estudio que se encuentran directamente entrelazados. En el presente documento se pretende esclarecer algunas de las principales relaciones generales que posibilitan este enlace. Así mismo, se busca puntualizar algunos tópicos relevantes que permitan afianzar una comprensión no reduccionista de sus relaciones.

Palabras clave: Cognición, tecnología, racionalidad, perspectiva de primera persona, perspectiva de tercera persona.

\begin{abstract}
Cognition, technology and rationality are directly bound and constitute three domains of study. In the present paper we pretend to clarify some of the main general relationships which allow such binding. Thus, it specifies some relevant topics to frame a non-reductionist comprehension of such relationships.

Key words: Cognition, Technology, Rationality, First-person perspective, Third-person perspective
\end{abstract}

\section{Resumo}

A cognição, a tecnologia e a racionalidade constituem três domínios de estudo que se encontram diretamente interligados. Este documento destina-se a esclarecer algumas das principais relaçóes gerais que possibilitam esta ligação. Também visa discutir alguns tópicos relevantes que permitam garantir uma compreensão não-reducionista de seus relacionamentos.

Palavras-chave: Cogniçáo, tecnologia, racionalidade, perspectiva de primeira pessoa, perspectiva de terceira pessoa. 

Carlos Mario Muñoz-Suárez: El proyecto de la Modernidad de construir una ciencia unificada y universal parece haber sido abandonado dada la hiperespecialización de muchas ciencias en el estudio de fenómenos particulares; no obstante, en las últimas décadas un gran puñado de problemas fundamentales ha obligado a que muchas de estas ciencias se unan para explicar fenómenos de nivel superior. Este es el caso de la antropología cognitiva, la neurociencia social y la etnotecnología. Otras ciencias se han unido para construir grandes sistemas y poder explicar una gran cantidad de fenómenos de distintas naturalezas; un ejemplo de esta unión es la teoría general de sistemas. ¿Considera usted que esta tendencia a la interdisciplinariedad nos conduce a repensar la racionalidad y, quizás, a la reconstrucción científica de una concepción de racionalidad?, o por el contrario, ¿considera que este auge de amalgamas disciplinares nos aleja del proyecto inicial de investigar la racionalidad?

Fernando Broncano: La interdisciplinariedad es una característica muy clara de la ciencia contemporánea. A veces ocurre que se comparten casi las mismas ecuaciones matemáticas, como ocurre con todo lo relacionado con fenómenos no lineales, a veces se converge en una zona de la realidad que necesita una perspectiva múltiple, como lo son los estudios sobre aspectos humanos. Este fenómeno de convergencia ha sido en realidad permanente a lo largo de la historia de la ciencia: la Química, por ejemplo, nació de un esfuerzo de este tipo. La transdisciplinariedad -más que la interdisciplinariedad, que supone que se conservan las anteriores disciplinas- es un fenómeno estructural de la ciencia que se irá mostrando cada vez más activo a medida que la especialización aumente. Se trata de hacer modelos de mundo usando materiales heterogéneos, y no creo que eso afecte a la racionalidad, al contrario, la convergencia de lo múltiple es parte de la cooperación científica. Es cierto que a veces se ha tendido a especificar la racionalidad en términos metodológicos que están tomados de tal o cual disciplina, pero son modelos de racionalidad muy superficiales. La racionalidad humana es unitaria, desde la ciencia al arte, desde la moral al conocimiento. Sería una locura pensar que hay racionalidades específicas, nos llevaría a la psicología del siglo XIX. La mente humana construye planes y modelos con materiales escasos y lo hace siguiendo sendas que son las mismas en todas las formas de creatividad.

C. $M .-S . .^{1}$ La distinción entre 'interdisciplinariedad' y 'transdisciplinariedad' ha representado un amplio tema de discusión en el ámbito de debate acerca de los

1 De ahora en adelante usaremos la abreviación C.M.-S. para referirnos al entrevistador -Carlos MuńozSuárez. 
recursos de conocimiento que debemos emplear en la explicación de fenómenos complejos, como las creencias propias de una cultura, las metarrepresentaciones, la conciencia etc. Al igual que las ciencias empíricas múltiples ramas de la filosofía se especializan constantemente, pero, en muchos casos, en la filosofía -sobre todo de enfoque analítico-, se especializan para dar como resultado el análisis conceptual ultra-delimitado de problemas explicativamente inaccesibles al tratamiento de ciencias empíricas. ¿Consideraría usted que la transdisciplinariedad puede ser pensada como una propiedad estructural que debe ayudarnos a tejer el diálogo entre filosofía y ciencia? ¿Qué tiene que cambiar en la ciencia para permitirnos este diálogo mancomunado?

F. $\boldsymbol{B} . \boldsymbol{:}^{2}$ Sí, la transdisciplinariedad es ya un elemento que no podemos dejar de lado: en casi todos los dominios de la ciencia se plantean convergencias hacia problemas y temas que exceden a las disciplinas tradicionales. La conciencia es uno de los ejemplos más claros. Dudo, sin embargo, que la filosofía pueda entrar como una más en el paquete: es una parte de la conversación de la humanidad, para usar la metáfora rortyana, pero su tempo es más lento que el de la investigación empírica. Está, más que nada, ahí para elaborar la experiencia que se está produciendo en el conocimiento o en la realidad. No obstante, no puede renunciar a un diálogo constante con las disciplinas implicadas, y sería una ceguera por parte de los científicos no tomarse en serio esta conversación.

C. $M$. - S.: ¿Considera usted que el flujo vertiginoso en el que instante tras instante se genera el cambio tecnológico nos compele a pensar que la conciencia, como un aspecto fundamental de nuestra mentalidad, ha de ser objeto de estudio preferentemente abordado experimentalmente? ¿Sería correcto soportar nuestras esperanzas de construir una ciencia acabada de la cognición y la conciencia -si eso fuese posible- a partir de nuestra confianza en el desarrollo tecnológico y técnico o, por el contrario, debemos pensar que el análisis conceptual y la reflexión metateórica nos llevara a la construcción de una teoría satisfactoria acerca de la cognición y la conciencia? ¿Son estas dos labores excluyentes?

F. B.: La ilusión de una ciencia humana es permanente en la cultura moderna desde Hume, así como la tensión entre las dos aproximaciones que señala: la empírica y la conceptual. Depende de si consideramos la humanidad como algo excepcional en la naturaleza o como parte del flujo natural de la vida. Mi

2 De ahora en adelante usaremos la abreviación F. B. para referirnos al entrevistado-Fernando Broncano. 
idea es que no podemos eliminar nuestra propia autodescripción en términos fenomenológicos y hermenéuticos sin socavar las propias investigaciones sobre la naturaleza humana. Pero eso no quiere decir que se pueda hacer antropología de sillón, o neurociencia o psicología: los datos experimentales sobre nuestra naturaleza son sorprendentes e inquietantes. Ahora bien, sólo quienes consideran que el trabajo científico y el filosófico están separados por barreras metodológicas o de otro tipo se preocupan por la cuestión de la tensión entre aproximaciones. El naturalismo, desde mi punto de vista, debería conformar la investigación filosófica, pero también el humanismo la investigación científica. Por ejemplo, figuras como Antonio Damasio están más allá de la distinción entre las dos culturas. El problema es a veces más de incultura que de división entre culturas.

\section{M.-S.: Me recuerda usted el libro Philosophical Foundations of Neuroscience} que recientemente ha puesto en jaque parte del capital teórico de la neurociencia actual. En esta obra sus autores proponían una visión conceptualmente cauta y radicalmente revisionista de las teorías actuales de la neurociencia, como los modelos de Damasio y otros. Este libro alimentó la idea de una brecha existente entre las necesidades de satisfacción explicativa-demandada por los filósofos-y la arquitectura teórica de la neurociencia actual, proponiendo cambios coyunturales que deberían ser enfrentados por los neurocientíficos. ¿Cuáles considera usted son casos recalcitrantes a tener en cuenta para ser superados de "incultura" en filosofía y en ciencia?

F. B.: Me resulta imposible responder a la pregunta de una forma escueta: el desconocimiento mutuo es abismal en todos los campos, pero en el tema que tratamos, diría esquemáticamente, que la experiencia humana es un horizonte normativo también para las neurociencias. Si al final no responden a las cuestiones de auto-entendernos de manera que se comunique lo que sabemos sobre el cerebro con lo que sabemos con nosotros como seres sociales, lingüísticos y técnicos, es que las preguntas quedan aún por responder, y sería una irresponsabilidad decir que esta experiencia es una ilusión de usuario creada por la "folk psychology". En ese caso podríamos responder que los proyectos neuro-computacionales son una ilusión de usuario creada por intereses académicos. En fin, es complicado.

C. M.-S.: Gran parte de la "industria" filosófica del siglo XX se ha puesto en marcha tomando como caballo de batalla, por un lado, el análisis conceptual y, por otro lado, la evidencia empírica. El problema de la naturalización de múltiples aspectos de nuestra vida mental surge constantemente como un reto 
a enfrentar tanto por las teorías filosóficas acerca del significado, del contenido mental conciente y de la causación intencional como por las teorías científicas de punta acerca de la cognición y la conciencia. A pesar del trabajo muchas veces mancomunado entre filósofos y científicos (neurocientíficos, ingenieros computacionales, psicólogos cognitivos etc.) no parece haber acuerdo con respecto a aquello que tratan de explicar cuando hablan de 'la mente conciente'; en este sentido, no parece haber acuerdo con respecto al explanandum ni consenso con respecto al proyecto de naturalización de la intencionalidad. ¿Desde su punto de vista qué teoría contemporánea acerca de la cognición y la conciencia considera que enfrenta mejor este reto?

F. $\boldsymbol{B}$.: Lo que nos ocurre es que el problema de la conciencia son muchos problemas en realidad: la vida consciente, la fenomenología, el autoconocimiento y autorreferencia, la autoidentidad, la agencia, la realidad del mundo consciente frente a los mecanismos subpersonales, etc. A mí me parece que deberemos pasar unos años de clarificación entre los varios problemas, y sobre todo de clarificación de las interdependencias entre las soluciones. La buena noticia es que la conciencia ha comenzado a ser un problema científicamente aceptable, algo que ha costado más de un siglo. Por otra parte, los filósofos han estado acostumbrados a pensar que la aproximación fenomenológica o lo contrario, el eliminacionismo, son las únicas alternativas, como si no hubiese una amplia zona de convergencia entre la fenomenología y la ciencia experimental. Mi actitud actual me lleva a ver con esperanza esta convergencia, en una línea en la que la fenomenología encarnada y la neurociencia pueden converger. Ciertamente, hay aspectos más complicados, particularmente los ligados a los aspectos hermenéuticos, puesto que la conciencia es un sistema que siempre está tratando de auto-interpretarse y auto-comprenderse, y esos aspectos superiores se encuentran en lo que llamamos conciencia narrativa, que en parte fue elaborada por algunos autores como Dennett como una forma de eliminacionismo, pero que creo que tienen una larga vida por delante si consideramos los relatos como un modo no puramente "intelectualista" de aproximación a la autoidentidad.

C. M.-S.: ¿Cuál considera que es el lugar de la experiencia conciente en el orden natural en tanto orden reconstruido conjuntivamente por las ciencias físicas?

F. B.: Hay aproximaciones funcionales que nos pueden dar una pista. En una descripción puramente causal del mundo ni siquiera podrían aparecer las funciones, y dudo que aún las singularidades y la historia contingente del uni- 
verso, lo que nos lleva a pensar que quizá acaso una descripción en términos de flujos de energía necesita complementarse con la presencia de la información, que, como es sabido, tiene problemas para ser reducida a puros paquetes de energía, puesto que es relativa o dependiente de respuesta de sistemas que ya son informacionales, sea como productores o como receptores. El problema es más bien para el Fisicismo clásico, que tiene grandes lagunas como descripción completa de la realidad, incluso en los niveles más básicos como la historicidad del cosmos, el origen de lo vivo, la naturaleza de los sistemas funcionales, etc. Lo que ocurre con la conciencia es que es un sistema muy difícil de explicar informacional o funcionalmente (¿cuál sería la función de la conciencia?), porque supone una especie social y técnica. Tengo esperanza, de todos modos, en que algunas convergencias entre neuropsicología, robótica y psicosociología puedan aproximarnos al problema duro de la conciencia.

C. M.-S.: En este sentido, ¿estaría de acuerdo en que enfrentar el problema duro de la conciencia -en el sentido de Nagel y Chalmers- radica en lograr explicar el conocimiento en primera persona que tenemos de nuestras propias sensaciones y experiencias y que, adicionalmente, este problema ha de ser la preocupación investigativa de un ámbito de conocimiento en formación entre la neuropsicología, la robótica y la psicosociología? ¿Necesitan estás disciplinas en sus múltiples convergencias modificar la formulación de este problema y, como muchos filósofos creen, convertirlo en un problema blando?

F. B.: No creo que sea tan simple: el conocimiento en primera persona debe ser él mismo sometido a escrutinio filosófico después de un siglo de estudios sobre la mente, sobre los sesgos, y sobre los mecanismos subpersonales, pero es cierto que hay un punto en el que tienen razón Nagel y Chalmers: al final, hay preguntas sobre la perspectiva en primera persona que no pueden ser eliminadas, y la noción de perspectiva no puede ser fácilmente funcionalizable como si fuera el lugar de una cámara grabadora. Perspectiva significa que en algún momento la conciencia debe suponer autoconciencia, o será simplemente consciencia animal.

C. M.-S.: En "Incompatibilidades en las teorías del contenido mental" (Broncano, 1999) usted diagnosticaba la tensión existente entre el representacionismo, el fisicismo y el holismo, concluyendo que había una profunda tensión aún no resuelta entre el principio de cierre causal del mundo físico, el principio de cierre del espacio de razones y el principio cierre de dependencia de procesos reales. ¿Cuál es 
su postura actual con respecto a esta incompatibilidad fundamental entre estos programas de investigación del contenido mental?

F. B.: Creo que es una de las tragedias filosóficas contemporáneas: no queremos renunciar a ninguno de los tres principios. Nuestra metafísica ya no puede ser excluyente, pero al mismo tiempo no nos es posible una actitud sincrética puesto que cada uno de los principios parece confrontarse con los otros. Hay una salida pragmatista, que es la de contemplar sólo las diferencias prácticas, en donde creo que la tensión es mucho menos, puesto que en la práctica tejemos los tres principios en modelos intermedios. La otra actitud, más complicada, pero que me parece más promisoria es intentar que las descripciones en términos causales, racionales o de sistemas sean ninguna de ellas cerradas, es decir, abandonar los tres principios a la vez, en una ontología abierta no necesitarista sino posibilista. Eso nos llevaría a abandonar las escisiones que crearon el romanticismo y la ontología del XIX entre el objetivismo y la comprensión del mundo. Esta actitud, sin embargo, exige un progresivo "consenso solapado", como diría Rawls, entre naturalistas y antinaturalistas. No espero sin embargo tanto de las divisiones actuales académicas como de la creciente necesidad de convergencia entre aproximaciones.

C. M.-S.: En Mundos Artificiales. Filosofía del cambio tecnológico usted defiende una noción de 'racionalidad' de acuerdo con la cual la racionalidad es una capacidad dependiente de nuestra astucia para "descubrir y aprovechar oportunidades" en este sentido, en sus propias palabras, "la racionalidad es [...] la propiedad que describe la calidad de los controles de calidad que adoptamos respecto de nuestras inferencias y decisiones" (Broncano, 2000 : 72), siendo una capacidad "fiable" en virtud del contexto en el que tomamos decisiones y, por tanto, histórico-socialmente "situada". Es un lugar común partir del concepto de racionalidad para reconstruir una noción teórica de realidad ( Searle, 1995) como algo que existe con total independencia de nuestra mente, de nuestras prácticas y de las culturas. ¿La noción de racionalidad que usted propone no nos guía más hacia el camino de lo que Nelson Goodman llamaba una "ontología evanescente" ${ }^{3}$, es decir, una visión de la realidad en la que entran en juego constante las convenciones, la historia, la sociedad etc. y en la cual aquello que

3 “[...] La estructura del mundo depende fuertemente del orden de los elementos y de la importancia comparativa de los géneros; la reordenación y el cambio de lugar del centro de interés, se encuentran entre los procedimientos más poderosos que se emplean para hacer y rehacer hechos y mundos..." (Goodman, 1995 : 187). 
designamos con la etiqueta 'mundo externo' se evanesce en la versión de mundo que soporta actualmente nuestras más profundas convicciones?

F. B.: Sí, está bien vista la cuestión. Yo no establecería la dicotomía entre estructura natural y convenciones, sino entre una ontología en la que la necesidad es la trama de la realidad y una ontología que permite las posibilidades. Las posibilidades están entre lo objetivo y lo subjetivo, necesitan una descripción ontológica en la que las singularidades sean relevantes. Dewey observaba que desde el punto de vista causal, la diferencia entre un ser vivo o muerto es inexistente, ambos son parte del flujo de energía. ¿Qué ontología, sin embargo, permite describir la vida y la muerte como hechos significativos de un organismo? No creo que la cuestión sea tanto de mundo externo/interno como de si en la descripción del mundo caben las singularidades. Otro ejemplo: cuando emprendemos un plan o una transformación agente de la realidad nos convertimos en iniciadores de una cadena causal; cuando culminamos un plan y obtenemos un resultado, éste evento adquiere una significación especial que ilumina todo el proceso anterior. Esta necesidad de incluir el sentido en el discurrir del mundo es lo que trata de capturar una idea de racionalidad como actualización de posibilidades, que es la que propongo. Es más una opción ontológica que epistemológica. Quizá deberíamos releer a Heidegger con ojos menos irritados por las divisiones académicas entre filosofía analítica y continental, y veríamos que la ontologización de la capacidad de comprender el mundo es una forma radical de plantearse el problema de la racionalidad.

C. M.-S.: ¿Esto no nos compele a pensar que la fenomenología y el análisis conceptual de la experiencia conciente son labores tan fundamentales en la construcción de una teoría metafísica como lo son la investigación básica propia de ciencias empíricas, como la química y la biología?

F. B.: Sí. Es más, me parece que es el centro de la investigación filosófica. Me atrevería a decir, con Dewey, que la elaboración de la experiencia humana es la tarea fundamental del filósofo, y que la experiencia humana es una especie de escala sobre la que deben medirse todos los resultados científicos o prácticos.

C. M.-S.: Usted recientemente defendió (Broncano, 1998) que la objetividad (en tanto resultado de múltiples tipos de prácticas de objetivación aceptadas) es un affordance. En este contexto, afirmó, adicionalmente, que la objetividad era una "suerte de invariancia ante el cambio de perspectiva" y que era aquello que 
nos permitía reidentificar objetos. De frente al clásico problema con el realismo metafísico ¿podríamos interpretar su postura como radicalmente revisionista de los presupuestos de esta postura?

F. B.: El realismo metafísico es hijo de la reacción filosófica a las prácticas de objetivación que definen la ciencia contemporánea. Se trata de encontrar una especie de descripción a priori que tenga una naturaleza intrínsecamente "objetiva", pero también podríamos pensar las cosas de otra manera, que es la de considerar que la objetividad es un logro de la mente humana para encontrar invariancias entre perspectivas, entre lenguajes, entre maneras de describir la realidad, entre subjetividades encontradas. Pensar la objetividad como un bien, no como una propiedad dada nos permite releer tanto la historia de la ciencia como la de la cultura en términos de un proyecto humano de conocimiento y lucidez. El realismo metafísico y el antirrealismo me parecen actitudes acomplejadas académicas ante la experiencia de objetivación del mundo de la ciencia. La idea de affordances -o estructuras objetivas de posibilidad- es una descripción alternativa que se escapa a la dualidad objetivismo/subjetivismo. Las estructuras de la subjetividad pueden ser tan objetivables como los procesos estocásticos, pero también hay que abrir la naturaleza para que sea habitada por espacios de posibilidad. Pongo el ejemplo de la pregunta anterior: la muerte, ¿Cómo ha de ser descrita, en términos objetivos o subjetivos?

C. M.-S.: ¿ Debemos encontrar medios explicativos para penetrar la brecha entre "conocimiento de primera persona" $\mathrm{y}$ "aproximaciones científicas en tercera persona» o es está dicotomía en sí misma aquello que debe ser disuelto bajo una nueva visión del orden natural?

F. B.: La tensión entre primera y tercera persona está por todas partes no sólo ni principalmente en la ciencia: es uno de los problemas filosóficos de primer orden. Es una brecha real que no puede ser resuelta intelectualmente, sino reconstruida en una experiencia abierta al otro y a la propia autodescripción extrańada. Yo diría que se trata de momentos distintos de un mismo proceso de autoconocimiento, más que de perspectivas incompatibles.

C. $M$. $-S .:$ Si, como usted sugiere, la identidad personal no es el resultado de prácticas de objetivación sino que es en el contexto de nuestras prácticas de discriminación y reidentificación en donde se experiencia dicha identidad ¿Qué podría decir frente aquellos (vid.: Perry, 1972) que sostienen que el problema de 
la identidad personal es un problema metafísico e independiente de cuestiones psicológicas?

F. B.: Las tres tradiciones metafísicas, la de la identidad como conciencia, la identidad como cuerpo y la de que la identidad no importa están equivocadas en lo que respecta a qué respuestas debe dar la metafísica. El problema metafísico de la identidad no es tanto el problema escolástico de la identidad de los particulares, que ha seguido siendo el trasfondo de la discusión académica desde Hume a Strawson cuanto la cuestión de la agencia como agencia autónoma: ¿̇soy yo quién está a cargo de la determinación del juicio o de la acción? $\mathrm{Y}$, en ese caso, ¿puedo ser descrito simplemente como un cuerpo, como una conciencia, como un flujo de estados mentales? También la objetivación debe llegar a la auto-objetivación. Un siglo de evidencias de autoengańo y akrasia, de pulsiones subpersonales y sesgos psicológicos nos ha hecho cautos respecto a la auto-transparencia pero también nos ha empeñado con más insistencia en un programa de autonomía real de la agencia. Y me parece que este es el lugar en el que hay que situar la pregunta por la identidad de las personas: ¿qué las convierte en singularidades causales?

C. M.-S.: Me hace usted pensar en la dicotomía clásica entre metafísica descriptiva y metafísica revisionista. Podríamos pensar que el encuentro de singularidades causales puede representar el surgimiento, o bien, de criterios contraintuitivos que debemos aceptar o de criterios incompatibles con los presupuestos metafísicos de las ciencias físicas básicas. En este sentido, ¿¿Son la metafísica descriptiva y la metafísica revisionista dos vías de conocimiento que se acoplan con nuestra perspectiva propia del mundo o debemos construir una nueva concepción de la metafísica?

F. B.: No acepto tan rápidamente el dilema. Me parece que el problema de las singularidades lo comparte la filosofía con la teoría de la relatividad, por ejemplo. La metafísica, de todas maneras, tiene que hacerse cargo del tejido de las dos imágenes, la científica y la cotidiana. Y hacerse cargo no significa eliminar una de las dos sino tejerlas.

C. M.-S.: Algunos investigadores han sostenido la idea de que la tecnología representa una brecha entre el hombre y los animales no humanos, ¿a partir de esto se puede establecer una distinción entre la mente animal y la mente humana? 
F. B.: La tecnología sí, la técnica no. La tecnología es hija del diseño científicamente orientado, mientras que la técnica es un modo de transformar el mundo basado en habilidades aprendidas. Somos animales técnicos como todos los simios, pero nosotros hemos aprendido a objetivizar las representaciones de los objetos posibles, a lo que he llamado técnicas de segundo orden: diseñar objetos para construir objetos, y esa capacidad depende de otra capacidad más básica, la capacidad de desacoplarse de la realidad y encontrar un territorio de imaginación y creatividad. Esa capacidad la dieron las sociedades complejas, basadas en patrones de acción objetivos (juegos, por ejemplo) y el lenguaje articulado, que modificó radicalmente tanto el cerebro como la socialidad.

C.M.-S.: Conociendo el abanico de sus intereses investigativos: ¡cuáles considera usted que son las principales tesis extraídas desde su análisis filosófico del cambio tecnológico que pueden iluminar la filosofía de la mente? o para una filosofo de la mente ¿qué aspectos de la filosofía de la tecnología pueden ser fundamentales?

F.B.: La idea de andamios culturales, es decir, de una cultura material construida por artefactos que se convierten en prótesis mentales me parece la parte más promisoria de mi trabajo. No es original: Daniel Dennett y Andy Clark siguen esta senda que, curiosamente, inició Heidegger. Mi aportación, me parece es la idea de entender los artefactos dentro de una ontología de la posibilidad, como nudos de relaciones y relatos objetivados, lo que hace que, a su vez, los artefactos sean ellos resultados de objetivaciones de lo imaginario. Me parece que así encontramos un lugar para la filosofía de la técnica como parte de la antropología. Me atrevería a insinuar que la antropología es lo más parecido a una filosofía primera en el sentido tradicional, algo distinto a los giros lingüístico y pragmático que están en la trama de la filosofía contemporánea.

C. M.-S.: En tanto filósofos, ¿¿debemos acoplarnos con un 'giro antropológico' que ilumine las múltiples convergencias con las ciencias empíricas y especiales? $\mathrm{O}$, en otras palabras, ¿Cuál ha de ser nuestra caja de herramientas en esta labor?

F.B.: No sé si hablaría de giro antropológico (la metáfora de los giros me produce mareo) sino de perspectiva: situar la experiencia humana en el núcleo normativo de la filosofía. La idea no es considerar ahora a los etnólogos como las nuevas autoridades, sino repensar cuál es el lugar del humanismo en el mundo contemporáneo. 
C.M.-S.: ¿Podría hablarnos acerca de sus proyectos actuales y cómo estos

rman un todo, si usted así lo considera, con su trabajo precedente o constituyen la plataforma de sus prospectos?

F.B.: Me encuentro en este lugar extraño de buscar en lo humano una dimensión que establezca una escala sobre todas las demás descripciones del universo. La reconsideración de las tradiciones antropológicas desde una visión que no sea la puramente estructural ni la puramente hermenéutica, desde una visión postdarwiniana, es la tarea que me ocupa actualmente. Por suerte, estamos en un buen momento, cuando ni el modernismo ni el posmodernismo son ya alternativas, ni la tradición analítica ni la continental. Se trata de, como señalaba Dewey, buscar una filosofía que haga más luminosa nuestra experiencia.

\section{Referencias}

Broncano, Fernando (1999). "Incompatibilidades en las teorías del contenido mental”, Teorema, Vol.: XVIII/2, Pp. 33- 48.

Broncano, Fernando (2000). Mundos Artificiales. Filosofía del cambio tecnológico, Paidós- UNAM, México D.F.

Broncano, Fernando (2008), Conferencia: "Representaciones, experiencias, prácticas y el giro agencial", Instituto de Investigaciones Filosóficas; UNAM; "Primer taller sobre Experiencia y Representación en la Ciencia", 25- 26 de Noviembre de 2008.

Goodman, Nelson (1995). De la mente y otras materias, Visor, Madrid.

Perry, John (1972). "Can the self divide?", Journal of Philosophy, Vol.: 69, Pp. 463- 488.

Searle, John (1995). The construction of social reality, The free press, New York. 
